\title{
New Approach for Range Measurements of Induced Magnetic Interactions in Pd
}

\author{
H. H. Bertschat, H. Granzer, H. Haas, R. Kowallik, S. Seeger, and W.-D. Zeitz \\ Bereich Festkörperphysik, Hahn-Meitner-Institut Berlin GmbH, D 14091 Berlin, Germany \\ and the ISOLDE Collaboration, CERN, Genève, Switzerland
}

(Received 1 August 1996)

\begin{abstract}
Induced long-range magnetic interactions in $\mathrm{Pd}$ single crystals covered with ferromagnetic $\mathrm{Ni}$ are observed in perturbed angular correlation measurements applying monolayer-resolved sample preparation. The ${ }^{100} \mathrm{Pd} /{ }^{100} \mathrm{Rh}$ probes sense magnetic interactions even seven atomic layers away from the Ni-Pd interface. The results are interpreted assuming fluctuations of magnetic moments strongly interacting with the ${ }^{100} \mathrm{Rh}$ nuclei. [S0031-9007(96)02061-3]
\end{abstract}

PACS numbers: 75.30.Hx, 75.70.Cn, 76.80.+y

The range of the magnetic polarization in the strongly exchange enhanced metal $\mathrm{Pd}$ in contact with $\mathrm{Ni}$ was investigated in a pioneering magnetometry experiment as early as 1984 [1]. As the most probable interpretation, the authors attributed the additional magnetic moment to $\mathrm{Pd}$, although they could not decide from the bare experimental results whether it is actually located in $\mathrm{Ni}$ or Pd. Meanwhile, the existence of ferromagnetic polarization in magnetic-layer systems with only a few monolayers (ML) $\mathrm{Pd}$ deposited on ferromagnetic substrates $(\mathrm{Fe}, \mathrm{Co}, \mathrm{Ni})$ is experimentally well established and theoretically treated [2]. The lattice parameters of such ultrathin Pd layers differ from the bulk value as a consequence of epitaxial growth. This can influence the magnetic properties considerably $[2,3]$. When the surface of Pd with bulk lattice parameters, e.g., of a single crystal, is covered with a film of a ferromagnetic material $(\mathrm{Fe}, \mathrm{Co}, \mathrm{Ni})$, neither the nature (static or fluctuating moments) nor the range of induced magnetic interactions are well known from experiments, since such a system is hardly accessible to many conventional experimental methods. Ab initio theoretical treatments of such systems are available, however [4].

The present experiment offers a new approach for range measurements of induced magnetic spin polarization in Pd. The further aim is to determine the nature of the polarization in Pd with minimized disturbance of the lattice parameters. Ideally, the only wanted disturbance is the magnetic influence by the ferromagnetic coverage; we have chosen isoelectric Ni. For a quantitative range measurement preferentially a monolayer-resolving method has to be applied. For this purpose the perturbed angular correlation (PAC) method with radioactive ${ }^{111} \mathrm{Cd}$ probe nuclei has recently been introduced [5]. We have selected ${ }^{100} \mathrm{Pd}$ decaying to ${ }^{100} \mathrm{Rh}$ as probe nuclei. The clean preparation and controlled deposition of radioactive ${ }^{100} \mathrm{Pd}$ probe atoms on $\mathrm{Pd}$ surfaces (avoiding other contaminations) were achieved within the present experiment [6]. Thus another transition-element probe (besides the Mössbauer probe ${ }^{57} \mathrm{Fe}$ ) is available for microscopic investigations of magnetic multilayer systems. ${ }^{100} \mathrm{Pd} /{ }^{100} \mathrm{Rh}$ as a PAC probe is of special importance, because it can be used as an extremely diluted impurity as well as a self atom. This opens the field for local investigations of surfaces and interfaces of $4 d$ elements or of composite $3 d / 4 d$-element multilayers.

In short the experiment is described as follows: ${ }^{100} \mathrm{Pd}$ atoms $\left(10^{-3}-10^{-4}\right.$ of a ML) were deposited on the surface of Pd and a number of ML of Pd and Ni was grown on top, consecutively. The short range of the hyperfine interaction provides ML resolution; the long penetration range of the gamma radiation allows measurements in any depth of the sample. The $4 d$-element probe ${ }^{100} \mathrm{Rh}$ can thus be used as a sensitive probe for local detection of a possible long-range magnetic interaction in Pd induced by the ferromagnetic Ni coverage. The surfaces of the single crystal $\operatorname{Pd}(111)$ samples (disks of $13 \mathrm{~mm}$ diameter and $0.5 \mathrm{~mm}$ thickness) were prepared by repeated argon sputtering and annealing at $1000 \mathrm{~K}$. The surface purity and structure were controlled in situ by Auger-electron spectroscopy and LEED. The ${ }^{100} \mathrm{Pd}$ activity $\left(T_{1 / 2}=3.6 \mathrm{~d}\right)$ was produced at the KFZ Karlsruhe, and chemically separated and purified at the HMI as described elsewhere [6]. It then was transferred into the UHV chamber ASPIC (Apparatus for Surface Physics and Interfaces at CERN; the chamber was located at the HMI during the construction of the new mass separator ISOLDE/CERN), base pressure of $2 \times 10^{-9} \mathrm{~Pa}$. By several steps of fractional evaporation [6] the activity was deposited on the Pd surface at $T=295 \mathrm{~K}$ and atomic layers of $\mathrm{Pd}$ and consecutively of $\mathrm{Ni}$ were grown on top of the radioactively marked Pd layer by molecular beam epitaxy, calibrated by medium-energy electron diffraction. The complete experiment was conducted without breaking the UHV.

The $\gamma-\gamma$ coincidences of the $84-75 \mathrm{keV}$ cascade in ${ }^{100} \mathrm{Rh}$ [7] were measured with four $\mathrm{BaF}_{2}$ detectors $\left(90^{\circ} / 180^{\circ}\right.$ arrangement) in a plane perpendicular to the sample surface. The isomeric intermediate nuclear state (spin $I^{\pi}=2^{+}$; lifetime $\tau_{N}=310 \mathrm{~ns}$; electric quadrupole moment $|Q|=0.151 \mathrm{~b} \quad[8]$; magnetic moment $\mu=$ $+4.23 \mu_{N}$ [9]) has a nuclear anisotropy coefficient of $A_{22}=0.175[7,10]$. Experimentally the $A_{22}$ coefficient is deduced from the ratio function of the count rates $(C)$

$$
R(t)=2\left[C\left(180^{\circ}\right)-C\left(90^{\circ}\right)\right] /\left[C\left(180^{\circ}\right)+C\left(90^{\circ}\right)\right],
$$


with $R(0)=\frac{3}{2} A_{22}^{\text {eff }}$ (finite detector angle effect taken into account in $\left.A_{22}^{\text {eff }}\right)$. The angular correlation is disturbed if electric field gradients (EFG) act on $Q$ or magnetic hyperfine fields $\left(B_{\mathrm{hf}}\right)$ on $\mu$ [10]. These static hyperfine interactions lead to an oscillating perturbation [11]. In the case of a static $B_{\mathrm{hf}}$, the time dependent count rates $C(t)$ are modulated involving the Larmor frequency $\omega_{L}=$ $g_{N} \mu_{N} B_{\mathrm{hf}} / \hbar$; no external magnetic fields were applied in our experiments. In the case of rapidly fluctuating magnetic interactions $\left(\ll \tau_{N}\right)$ no oscillating perturbation pattern will be measured and the fit function for Eq. (1) can be written as $R(t)=\frac{3}{2} A_{22}^{\text {eff }} \exp \left(-\lambda_{D} t\right)$, i.e., only a reduction of the anisotropy with time is observed. According to Abragam and Pound [12] $\lambda_{D}$ is given in the simplest case as

$$
\lambda_{D}=\tau_{c} \omega_{s}^{2} 2 S(S+1),
$$

with $\omega_{s}$ being proportional to the interaction energy between the ionic spin $S$ of the probe and the nuclear moment and $\tau_{c}$ the relaxation time of the electronic spin regarded as a correlation time.

The PAC time spectra, according to Eq. (1), are collected in Fig. 1. Figure 1(a) shows the result for the nonmagnetic sample where on top of the $\operatorname{Pd}(111)$ surface, doped with $10^{-3} \mathrm{ML}$ of ${ }^{100} \mathrm{Pd}, 3 \mathrm{ML}$ of $\mathrm{Pd}$ were grown. Because of the cubic symmetry in fcc $\mathrm{Pd}$, a constant $R(t)$ is expected; the slight damping can be caused by a broad EFG distribution due to nonperfect layer growth, but obviously the majority of probe atoms is located on regular lattice sites. The spectrum in Fig. 1(b) was obtained after covering the above system with $5 \mathrm{ML}$ of $\mathrm{Ni}$. $\mathrm{Ni}$ of this thickness is ferromagnetic at $T=295 \mathrm{~K}$ [13]. The $R(t)$ function decays rapidly within $<5 \mathrm{~ns}$ to zero and no oscillations are observed, in contrast to bulk $\mathrm{Ni}$, Fig. 1(c). Thus ${ }^{100} \mathrm{Rh}$ serves as an indicator for an induced long-range magnetic interaction in Pd. For the decision whether these induced interactions are of static character (very high $B_{\mathrm{hf}}$ producing Larmor frequencies beyond the electronic time resolution) or of dynamic character, according to Eq. (2), we have performed two further experiments. In order to examine the possibility of static fields in thick $\mathrm{Pd}$, the ${ }^{100} \mathrm{Pd}$ ions were positioned $7 \mathrm{ML}$ away from a $2 \mathrm{ML}$ coverage of $\mathrm{Ni}$ with a lower $T_{C}$. At this depth in $\mathrm{Pd}$ a possible static $B_{\mathrm{hf}}$ at the ${ }^{100} \mathrm{Rh}$ probe nuclei should be considerably reduced, especially close below $T_{C}$. No magnetic interactions are observed at $295 \mathrm{~K}$ [Fig. 1(d)]. A reduction of $R(t)$ with temperature starts at $T \approx 150 \mathrm{~K}$. No oscillating perturbations are observed at any temperature [Fig. 1(e)], but the effective nuclear anisotropy is increasingly reduced with decreasing temperature until at $90 \mathrm{~K}$ the time spectrum in Fig. 1(f) [indistinguishable from 1(b)] is obtained. This behavior was reversible, i.e., at elevated temperatures we again measured spectra like the one in Fig. 1(d) in the same sample. In Ref. [1] the magnetic moment of the whole $\mathrm{Ni} / \mathrm{Pd}$ sample was measured, and its increase was

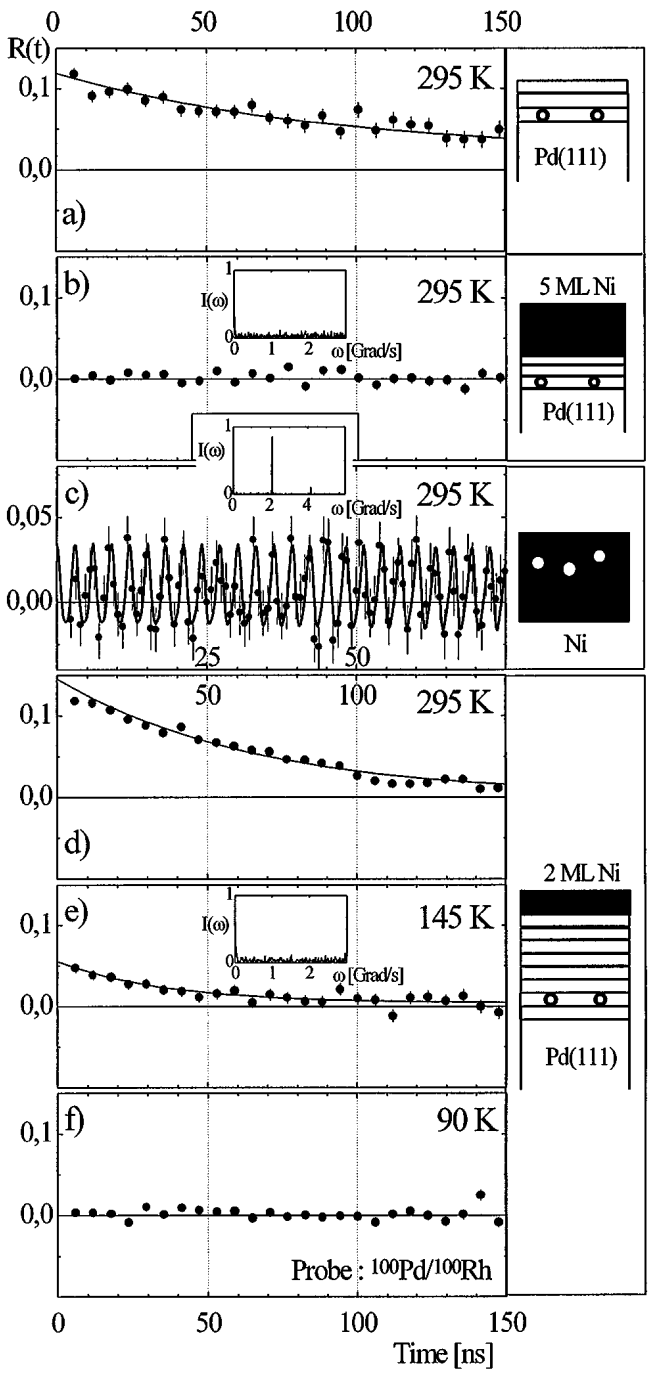

FIG. 1. PAC time spectra of ${ }^{100} \mathrm{Pd} /{ }^{100} \mathrm{Rh}$, positioned in $\mathrm{Ni} / \mathrm{Pd}$-layer systems as sketched on the right hand side of each spectrum. All spectra were taken with an electronic time resolution of $1.0 \mathrm{~ns}$. For graphical reasons the data are compressed. Solid lines in (a) and (d) are exponential fits accounting for broad distributions of weak EFG's (see text). In order to keep the ${ }^{100} \mathrm{Pd}$ probe atoms in position no annealing was performed. In the data of (b), (e), and (f) no oscillations could be detected. For comparison, our PAC spectrum with magnetic oscillations for ${ }^{100} \mathrm{Rh}$ in the bulk of $\mathrm{Ni}$ is shown in (c) (different scales) corresponding to the known value of $B_{\mathrm{hf}}=20.2 \mathrm{~T}$ [22]; the fit is taken from Eq. (1) including the Larmor frequency $\omega_{L}$ [23]. In (b), (c), and (e) Fourier transforms (in Grad/s) of the raw data are inserted.

attributed to the formation of moments in the added $\mathrm{Pd}$ layers. With our local investigation we found the proof that there is induced magnetic interaction in $\mathrm{Pd}$ up to at least seven atomic layers away from the Ni coverage. In order to explain the reduction of $R(t)$ by a static $B_{\mathrm{hf}}$ one has to assume extremely high $B_{\mathrm{hf}}$ values $\gg 30 \mathrm{~T}$ (with a broad distribution) which is not supported by any moment calculations [3,14]. We consider the development in time of $R(t)$ as a result of fluctuating ${ }^{100} \mathrm{Rh}$ moments caused 
by induced magnetic interactions. For the relaxation rate $\lambda_{D}$ we can give a lower limit due to the time window of our experiment. From the spectra in Figs. 1(b) and 1(f) we obtain $\lambda_{D}\left({ }^{100} \mathrm{Rh}\right)>1 \times 10^{9} \mathrm{~s}^{-1}$ for the magnetic $4 d$ probe ${ }^{100} \mathrm{Rh}$ [Eq. (2)].

A complementary experiment was performed with the nonmagnetic $s p$-element $\mathrm{Cd}\left(\mu=-0.765 \mu_{N}\right.$ [7]) positioned in Pd as in Figs. 1(a) and 1(b). This experiment was performed in ASPIC at the mass separator ISOLDE/CERN [15] with the PAC probe ${ }^{111 m} \mathrm{Cd} /{ }^{111} \mathrm{Cd}$ using a similar procedure as described elsewhere [16]. The results are shown in Fig. 2: No magnetic oscillation of ${ }^{111} \mathrm{Cd}$ and thus no static magnetic hyperfine field is observed at this probe position in thick Pd. Consequently, a possible hypothesis that intermixing of the interfacial layers during the evaporation of $\mathrm{Ni}$ on $\operatorname{Pd}(111)$ causes a (ferromagnetic) alloy is not supported by this result. Furthermore, a possible static $B_{\mathrm{hf}}$ would be less than $0.01 \mathrm{~T}$, considerably smaller as expected from the values $B_{\mathrm{hf}}(\mathrm{Cd})=7 \mathrm{~T}$ in bulk $\mathrm{Ni}$ and $B_{\mathrm{hf}}(\mathrm{Cd})=4 \mathrm{~T}$ in one $\mathrm{ML}$ of Pd on Ni [17] [compare Fig. 2(c)] along with moment calculations [14]. We conclude that already in the third ML of Pd the moments of the Rh probes [Fig. 1(b)] are fluctuating rather than their nuclei being subject to strong static magnetic hyperfine fields. Aspects of fluctuating magnetic moments in Pd were discussed in Ref. [3] for Fe-Pd-Fe trilayers with Pd exceeding 4 ML.

In the interpretation of the results we regard paramagnetic $\mathrm{Pd}$ as a strongly exchange enhanced metal never reaching a Curie temperature. From earlier PAC experiments [18,19], applying external magnetic fields, it is known that in pure paramagnetic Pd the local mo-

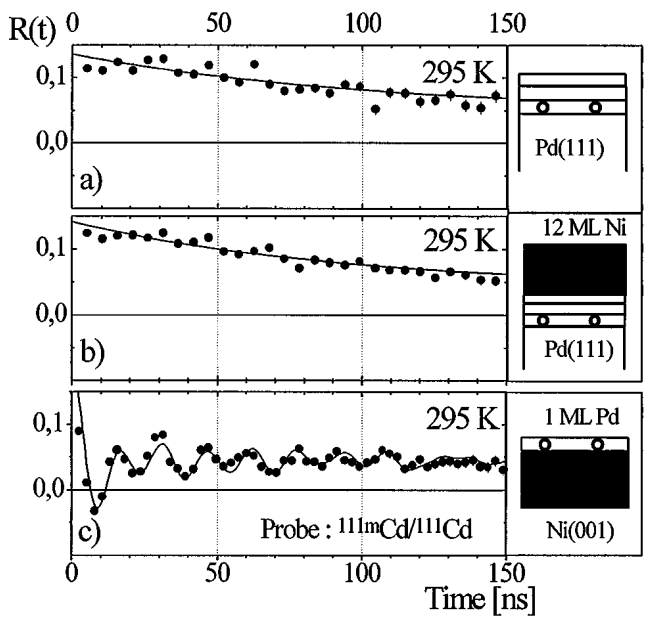

FIG. 2. PAC time spectra of ${ }^{111 m} \mathrm{Cd} /{ }^{111} \mathrm{Cd}$ [16] in $\mathrm{Pd}$ as indicated on the right; compare Figs. 1(a) and 1(b). No static magnetic hyperfine fields causing oscillations are observed at the $s p$-element $\mathrm{Cd}$, when thick $\mathrm{Pd}$ is covered by $12 \mathrm{ML}$ of Ni. For comparison, a PAC spectrum of static magnetic (and electric) hyperfine interactions of ${ }^{111} \mathrm{Cd}$ positioned in one ML $\mathrm{Pd}$ on a Ni single crystal in zero external field is shown in (c), taken from Ref. [17]. ment of $\mathrm{Rh}$ probes (interacting with their nuclear magnetic moment) is rather unstable with a high spin fluctuation temperature of $220 \mathrm{~K}$. The correlation times $\tau_{c}$ are too short to affect the nuclear alignment and no damping of $R(t)$ was detected $\left(\lambda_{D}<2 \times 10^{5} \mathrm{~s}^{-1}\right)$. Recently it was shown that the spin fluctuation rates of $\mathrm{Rh}$ in $\mathrm{Pd}$ are strongly suppressed in the presence of diluted polarizing magnetic impurities $(P d \mathrm{Fe}$ above $T_{c}$ ) [19]. Furthermore, the nature of the interaction between the ${ }^{100} \mathrm{Rh}$ impurities and the polarized Pd host via the $d-d$ interatomic exchange interaction was discussed comprehensively. We apply this knowledge to the present experiment and write Eq. (2) explicitly as $\lambda_{D}=2\left(\mu_{N} / \hbar\right)^{2} g_{N}^{2} B(0)^{2} \tau_{c}(S+1) / S\left[\mu_{N}\right.$ : nuclear magneton, $g_{N}$ : nuclear $g$-factor, $B(0)$ : magnetic hyperfine field at $T=0 \mathrm{~K}$ ] obtaining a spin fluctuation rate $\tau_{c}^{-1}$ included in the compilation of Table I. In addition, for Ni above $T_{C}$ we show the rates of ${ }^{100} \mathrm{Rh}$, taken from Ref. [20]. In Ref. [20] also a comparison to the spin fluctuation rates, as they are obtained in neutron diffraction experiments in Ni $\left(T>T_{C}\right)$, was attempted; in Table I we have inserted such a result using more recent data [21]. From this compilation it can be seen that the value for the $\mathrm{Ni}$ $\mathrm{Pd}$ interface is extremely small. We compare $\mathrm{Pd}$ with $\mathrm{Ni}$ in the ferromagnetic and paramagnetic phases. In contact with $\mathrm{Ni}$, Pd "borrows" the $T_{C}$ from ferromagnetic $\mathrm{Ni}$ when only a few ML of Pd are grown on Ni. The spin-aligned $\mathrm{Ni}$ ions try to align the Pd ions by exchange interaction and the conduction-electron polarization succeeds in a static alignment of the Pd moments $[1,17]$. If $\mathrm{Pd}$ is thicker, as in our experiments with a single crystal, the aligned Pd spin ensemble becomes larger but unstable against the alignment with $\mathrm{Ni}$. We obtain (presumely collective) extraordinarily slow fluctuations of the magnetic moments (deduced from our measurement of $\tau_{c}^{-1} \leq 1 \times 10^{10} \mathrm{~s}^{-1}$ ) at least in the range of three up to seven atomic layers away from the ferromagnetic Ni. They are comparable to the fluctuations in $\mathrm{Ni}$ above $T_{C}$ $[20,21]$, where the spin clusters become larger and the fluctuations slower, the closer $T_{C}$ is approached. In our $\mathrm{Ni}$-Pd system the spin clusters might be even considerably larger, which seems plausible in a layer system for geometrical reasons. Additionally, there is the essential difference that in the Ni-Pd system a preferential

TABLE I. Selected paramagnetic spin fluctuation rates.

\begin{tabular}{lcc}
\hline \hline \multicolumn{1}{c}{ System } & $\tau_{c}^{-1}\left[\mathrm{~s}^{-1}\right]$ & Ref. \\
\hline${ }^{100} \mathrm{Rh}$ in $\mathrm{Ni} / \mathrm{Pd}^{\mathrm{a}}$ & $\leq 1 \times 10^{10}$ & \\
${ }^{100} \mathrm{Rh}$ in $\mathrm{Pd}($ pure $)$ & $4 \times 10^{13}$ & {$[18,19]$} \\
${ }^{100} \mathrm{Rh}$ in Pd $(2 \% \mathrm{Fe}, T=120 \mathrm{~K})$ & $2 \times 10^{12}$ & {$[19]$} \\
${ }^{100} \mathrm{Rh}$ in Ni $\left(T_{C}+0.1 \mathrm{~K}\right)^{\mathrm{b}}$ & $3 \times 10^{12}$ & {$[20]$} \\
Neutron scattering in Ni $\left(T_{C}+30 \mathrm{~K}\right)^{\mathrm{b}}$ & $3 \times 10^{11}$ & {$[21]$} \\
\hline \hline
\end{tabular}

${ }^{\mathrm{a}} B(0)=20 \mathrm{~T}$ was taken as in [19].

${ }^{\text {b}}$ Measurements closest to $T_{C}$ are taken. 
orientation with respect to the static Ni moments remains. In the first few Pd layers, which are close to the ferromagnet, the net magnetic moments add (due to the preferential orientation) to the magnetic moment of the ferromagnetic layer, keeping our experiment in agreement with Ref. [1].

Calculations on either $\omega_{s}$ or $\tau_{c}$ [Eq. (2)] could yield deeper insight into the fluctuation process.

The authors are indebted to D. Forkel-Wirth and J.P. Lohmüller for their help in the experiments at ISOLDE. We thank K.-H. Bennemann, P. H. Dederichs, and F. Mezei for encouraging discussions. This work was performed in partial fulfillment of the requirements for a doctorate in science (H.G. and S. S.). It was supported by resources of the Kernforschungszentrum Karlsruhe $\mathrm{GmbH}$. The ${ }^{100} \mathrm{Pd}$ nuclide production was conducted by K.-H. Assmus.

[1] U. Gradmann and R. Bergholz, Phys. Rev. Lett. 52, 771 (1984); J. Mathon, J. Phys. F 16, 669 (1986).

[2] U. Gradmann, in Handbook of Magnetic Materials, edited by K.H.J. Buschow (Elsevier S.P., Amsterdam, 1993), Vol. 7, p. 1; B. Heinrich and J. F. Cochran, Adv. Phys. 42, 523 (1993).

[3] E. E. Fullerton, D. Stoeffler, K. Ounadjela, B. Heinrich, Z. Celinsky, and J. A.C. Bland, Phys. Rev. B 51, 6364 (1995); Z. Celinski, B. Heinrich, J.F. Cochran, W.B. Muir, A. S. Arrott, and J. Kirschner, Phys. Rev. Lett. 65, 1156 (1990).

[4] S. Blügel, B. Drittler, R. Zeller, and P. H. Dederichs, Appl. Phys. A 49, 547 (1989).

[5] J. Voigt, X. L. Ding, R. Fink, G. Krausch, B. Luckscheiter, R. Platzer, U. Wöhrmann, and G. Schatz, Phys. Rev. Lett. 66, 3199 (1991).

[6] S. Seeger, H. Haas, and A. Mayer, Hyperfine Interact. 84, 211 (1994); S. Seeger, Ph.D. thesis, FU, Berlin, 1993. In order to avoid the very difficult chemical separation and purification procedures, efforts are made to obtain the
${ }^{100} \mathrm{Pd}$ activity directly from a mass separator, either at the ISOLDE/CERN, Geneva or at the ISL/HMI, Berlin.

[7] C. M. Lederer and V. Shirley et al., Tables of Isotopes (John Wiley, New York, 1978).

[8] H. Haas, A. Carbonari, and P. Blaha, Annual Report No. HMI-B, 1994, Vol. 526, p. 24.

[9] E. Matthias and D. A. Shirley, Nucl. Instrum. Methods 45, 309 (1966).

[10] H. Frauenfelder and R. M. Steffen, in Alpha-, Beta-, and Gamma-Ray Spectroscopy, edited by K. Siegbahn (NorthHolland, Amsterdam, 1965), Vol. 2, p. 997.

[11] H.-E. Mahnke, Hyperfine Interact. 49, 77 (1989).

[12] A. Abragam and R. V. Pound, Phys. Rev. 92, 943 (1953).

[13] Yi Li and K. Baberschke, Phys. Rev. Lett. 68, 1208 (1992); K. Baberschke, Appl. Phys. A 62, 417 (1996).

[14] J. Mathon, Rep. Prog. Phys. 51, 1 (1988); P. H. Dederichs, P. Lang, K. Willenborg, R. Zeller, N. Papanikolaou, and N. Stefanou, Hyperfine Interact. 78, 341 (1993).

[15] E. Kugler, D. Fiander, B. Jonson, H. Haas, A. Przewloka, H. L. Ravn, D. J. Simon, and K. Zimmer, Nucl. Instrum. Methods Phys. Res., Sect. B 70, 41 (1992).

[16] E. Hunger and H. Haas, Surf. Sci. 234, 273 (1990).

[17] With only 1 or 2 ML of Pd on thick Ni, distinct oscillation frequencies of ${ }^{111} \mathrm{Cd}$ in $\mathrm{Pd}$ caused by static $B_{\mathrm{hf}}$ 's (and EFG's) were measured at ISOLDE; H. Granzer, H.H. Bertschat, H. Haas, W.-D. Zeitz, J.P. Lohmüller, and G. Schatz, experiment in progress.

[18] G. N. Rao, E. Matthias, and D. A. Shirley, Phys. Rev. 184, 325 (1969).

[19] S. Khatua, S. N. Mishra, S. H. Devare, and H. G. Devare, Phys. Rev. Lett. 68, 1038 (1992).

[20] A. M. Gottlieb and C. Hohenemser, Phys. Rev. Lett. 31, 1222 (1973).

[21] P. Böni, H. A. Mook, J. L. Martinez, and G. Shirane, Phys. Rev. B 47, 3171 (1993).

[22] S. Koički, T. A. Koster, R. Pollak, D. Quitmann, and D. A. Shirley, Phys. Lett. 32B, 351 (1970).

[23] E. Matthias, S. S. Rosenblum, and D. A. Shirley, Phys. Rev. Lett. 14, 46 (1965). 